\title{
COVID and African Americans: Addressing Disparities in Mental Health
}

\author{
James Maiden, Ed.D. ${ }^{1}$, Phronie Jackson, Ph.D. ${ }^{2}$, Nathalie Mizelle, Ph.D. ${ }^{3}$ \\ ${ }^{1}$ Assistant Dean of Administration, University of the District of Columbia \\ ${ }^{2}$ Assistant Professor of Health Education, University of the District of Columbia \\ ${ }^{3}$ Associate Professor of Rehabilitation Counseling, University of the District of Columbia
}

\begin{abstract}
The mental health implications stemming from the ongoing COVID-19 pandemic are still to be revealed. However, research suggests the outlook is quite concerning. The COVID-19 pandemic effect on communities of color has provided more attention and respect to African Americans' need for mental health care. Racism worsens the adverse impacts of social determinants of health, causing health disparities for African Americans. This conceptual article explores COVID19 and racism disproportionately affecting African Americans' mental health and psychological well-being. The article also provides recommendations for mental health professionals to offer advocacy and to combat the problem.
\end{abstract}

KEYWORDS: mentalhealth, racism, psychological distress, stigmatization

\section{INTRODUCTION}

Despite improvements in reducing the health disparity gap in the United States, African Americans still fare far worse than their White peers across almost every health indicator, especially in mental health (Noonan, 2016). Mental illness affects millions of people in the United States but unfortunately afflicts African Americans more drastically than others. Buser (2009) posited that African Americans struggle with mental illness and deal with more persistent mental health issues than Whites. With the impact of the COVID-19 and the emergence of new variants on the African American community, mental health has been given more consideration. The pandemic has underpinned the fact that every individual's health is intertwined with others' health in their community. However, due to racial and ethnic disparities, African Americans are suffering a disproportionate impact and shouldering the burden of the COVID-19 pandemic (Gibbs et al., 2020).

COVID-19 has impacted African American lives, including their mental health, at a higher rate than other racial/ ethnic groups (Ibrahimi et al., 2020). The pandemic has exposed deep-seated inequalities in mental health care for African Americans and magnifies social and economic issues contributing to poor health outcomes, compounding longstanding racial disparities (Mizelle et al., 2020). The authors support that COVID-19 has impacted African Americans more than their counterparts. To this end, the article will discuss recommendations for counselor educators and mental health professionals.

\section{LITERATURE REVIEW}

The COVID-19 pandemic has further exposed the distressful reality of racial disparity in the United States. African Americans are burdened with morbidity and mortality due to COVID-19 (Mizelle et al., 2020). African Americans are the second-largest racial minority, comprising an estimated $13.4 \%$ of the population, but they comprise 30 percent of COVID-19 cases (Getachew, 2020). Individuals who work in essential jobs or reside in high-density housing are more likely to contract the virus because of unavoidable personto-person interaction. Those with less access to quality health care and certain chronic diseases have more severe outcomes. These conditions for greater exposure and worse outcomes have been concentrated in communities of color due to decades of deliberate policy choices and racist institutional practices like systemic housing discrimination of Black families. This has contributed to African Americans being infected and dying from COVID-19 at more than 1.5 times their share of the population (Getachew, 2020).

The COVID-19 pandemic has also created widespread mental health problems. Recent coronavirus survey data from the U.S. Census Bureau show that 41 percent of U.S. adults reported symptoms of anxiety and depressive disorders, compared to 11 percent in 2019. (U.S. Census Bureau, 2020) It was also reported that African Americans were 
more likely to report symptoms. The pandemic's health and economic consequences have a detrimental effect on mental health, particularly among African Americans (Ibrahimi et al., 2020). Gould \& Wilson (2020) revealed that African American adults are $20 \%$ more likely to experience mental health issues compared with the rest of the population.

Racism intensifies the harmful impacts of social determinants of health, causing racial health disparities for African Americans. African Americans are more susceptible to contract COVID-19 due to systemic Racism that historically carved out the type of work they are obliged to perform, including deliberate neighborhood and school segregation policies that currently explain their increased workplace and environmental exposure to COVID-19 infection. As a result, a more significant proportion of African Americans now suffer from the stress, anxiety, and depression engendered by COVID-19 (Mizelle et al., 2021).

The fear of getting infected by COVID-19 has caused mental distress for African Americans. African Americans and their families are highly represented among essential workers and have suffered more family and community member deaths. Gibbs et al. (2020) suggested that African Americans are three times as likely to know someone who has died from COVID-19 than Whites. The loss of family and community members, compounded by social distancing measures, acts as a stressor by limiting access to support systems. These stresses and losses increase the risks of depression, anxiety, substance use, and suicide, as well as poor physical health (Mizelle et al., 2021).

\section{Origins of Disparity}

The health disparities that exist for African Americans are not random phenomena, but instead, they are byproducts of a system of events, circumstances, and variables consistently interacting. There are three significant barriers to health care that affect all Americans: lack of health insurance, lack of a usual source of care, and perception of need (Cheathman et al., 2008). When these factors are missing or neglected, health is affected. Unfortunately, these factors are neglected more in African American communities (Plowden, 2003). These factors and others are responsible for the racial disparity in mental health treatment and health-seeking. The disparity in mental health seeking and treatment has been attributed to alternative coping strategies and African Americans' attitudes towards services (Buser, 2009). Alternative coping mechanisms/resources used in African American communities can be why some are hesitant to seek mental health treatment. These personal and cultural coping resources may serve as substitutes for professional mental health assistance and affirm social norms regarding the irrelevance of such services. African Americans rely heavily on family and friends' encouragement rather than directly asking for professional assistance. Another alternative resource African Americans use is religious leaders. Research has found that African Americans report seeking assistance with personal problems from religious leaders to a higher degree than they seek help from mental health professionals. Similar research found that African American churches provided more mental health services than Caucasian churches. This affects those individuals that seek mental health treatment because individuals who initially sought assistance from clergy for issues/concerns were less likely to seek mental health services (Buser, 2009).

\section{COVID 19 and The Social Determinants of Health}

Economic stability, Education and Health Care access and quality, Neighborhood and Built Environment, and Social and Community Context are the five domains characterized as the social determinants of health (SDOH). They are the environmental circumstances that influence a broad span of health, functioning, and quality-of-life risk and outcomes. $\mathrm{SDOH}$ are the non-medical factors such as where individuals age, are born, learn, live, play, work, and worship (Healthy People 2030, 2022). Additionally, SDOH determines opportunities that can impact individual choices, health, and behaviors (CDC, 2021). Historically SDOH have disproportionately affected communities of color, particularly African American communities (Noonan et al., 2016).

According to Kirby (2020), a considerable effect on COVID19 outcomes stems from many of the SDOH not limited to race and ethnicity and inclusive of smoke exposure and homelessness. In the last third of 2021, the Economic Impact of COVID-19 was more devastating for African Americans than Whites, with $68 \%$ of Blacks reporting difficulty paying household expenditures compared to $45.7 \%$ of White adults (U.S. Census, 2020). In a social context, a Washington Post poll shows that one-third of African American people know someone who has died from COVID-19 (Goldstein and Guskin, 2020). Also, the Associated Press reports that Blacks are two to three times more likely than Whites to die from COVID19 (Johnson et al., 2021). This has had a significant impact on the health and well-being of African Americans and their overall social cohesion of the African American community, including but not limited to relationships and interactions with family, friends, co-workers, and community members. The urban neighborhood and built environment often result in unfavorable elements such as crowding, poverty, and racism, especially in African American communities have contributed to the higher rates of COVID-19 with that population (Frumkin, 2021). Participants of a survey conducted by Jackson et al. (2021) responded that healthcare access was difficult. Approximately 19\% of the respondents reported not being able to receive dental care during the Washington, DC COVID-19 stay-at-home order. The study also demonstrated how access to quality education was disrupted, as $13 \%$ of the participants reported either their or their children's education was halted or transitioned to a virtual delivery. The study also addressed the impact of COVID-19 on mental health. Seventeen and fifteen percent of 
the respondents reported mental and emotional impacts on their health and well-being. These data support the suggestion that more attention and respect to African Americans' need for mental health care. As such, intervention to increase awareness, knowledge, and skills that promote mental wellbeing, such as self-care practices and Mental Health First Aid training, is recommended.

\section{Psychological Distress and Access to Care}

The effect of the COVID-19 pandemic is another area of disparities compared to white individuals that existed before the pandemic. Psychological distress for many African Americans often goes untreated. For example, Gibbs et al. (2020) reported that 69\% of African Americans with mental illness and $42 \%$ of African Americans with serious mental illness received no treatment in 2018. Similarly, 88\% of African Americans with substance use disorders reported receiving no treatment in 2018. Before the pandemic, individuals with mental illness faced many obstacles in obtaining adequate physical and mental health care. While stigma and minimizing symptoms are often identified as significant obstacles for mental health care, studies have shown that cost is cited as the most significant barrier; thus, this most impacts poor and historically oppressed African American communities (Gibbs et al., 2020).

Problems with pandemic-related access to care also impact African American community with psychological distress. They have limited access to technology for participating in telehealth and distance learning. An unintentional consequence of distance learning further exacerbates the gap between African Americans and their counterparts due to a significant technological gap. For example, not having Wi-Fi access adversely affects distance learning and access to telehealth. Additionally, Pierce and Stevermer (2021) reported African Americans were less likely to use telehealth, and when telehealth was used, it was more often limited to audio capabilities alone. Their findings suggested that disparities worsened existing racial disparities for health outcomes among racial and ethnic minority populations.

\section{Stigmatization}

Cultural aspects must be considered when examining the attitudes of African Americans toward mental health services. Stigma is often connected with mental illness, but this is especially true in the African American community (Mizelle et al., 2020). African Americans have been shown to hold more negative attitudes toward mental health and mental illness than White Americans (Cruz et al., 2008). In the African American community, stigma in the family and community plays a role in dissuading African Americans from seeking mental health care (Buser, 2009). Individuals are fearful of being associated with mental health treatment or mental illness. Research has shown that employers are less likely to hire individuals with a known mental illness, and landlords are less likely to lease to individuals for the same reason (Strauser et al., 2009). Buser (2009) confirmed that African Americans identified embarrassment, disgrace, and isolation as associated with mental illness and seeking mental health treatment.

\section{Mistrust}

The history of racial discrimination has contributed to African Americans' psychological disposition to mistrust the healthcare system (Cokley et al., 2021). This mistrust has led to an under-representation of African American participants in research trials, which has taken on a new urgency as the world faces this generation's most significant public health challenge. From a historical perspective, several studies have found a legacy of Racism in medicine. The Tuskegee Syphilis Study, a clinical trial conducted with African American subjects who were denied appropriate treatment opportunities, is a crucial factor underlying African Americans' distrust of medical and public health interventions, including vaccination (Gamble, 1997).Current research highlights cultural mistrust of the healthcare system. Scharff et al. (2015) conducted a qualitative study that explored barriers to healthcare participation among African Americans. The participants revealed that mistrust remains a critical barrier to healthcare and research. These studies are significant in understanding African Americans' hesitancy and distrust of the COVID-19 vaccine.

Thedifferentiationinmentalhealth diagnosisand prescription patterns have also contributed to African Americans' mistrust towards the mental health system. There has been evidence that African Americans are more likely to be diagnosed with schizophrenia and paranoid personality disorder than Whites. Buser (2009) postulated that White clinicians exhibited this pattern to a greater degree. Racial bias may be a part of these diagnosis differences, and clinicians may hold racial stereotypes about mental illness. Provider bias in treating mental disorders has long been a deterrent for African Americans and their tendency to seek care (Diala et al., 2001). The issue of biased care can explain the different prescription and diagnostic rates. Studies have shown that African American clients were less likely to be prescribed atypical antipsychotics. This is significant because atypical antipsychotics are believed to produce more beneficial effects (Buser, 2009). The continued mistrust of mental health diagnosis and prescription patterns combined with the COVID 19 pandemic could prevent African Americans from seeking assistance to support their health and wellness.

\section{A CALL TO ACTION}

The pandemic has exacerbated the mental health disparities that impactsAfrican Americans. Mental health care providers must partner with affiliations that promote mental health for the African American community (Mizelle et al., 2021). Research has found that African Americans are more likely to seek mental health assistance from religious leaders than mental health professionals (Buser, 2009). With this 
knowledge, counselors and mental health professionals should advocate and work with community churches and religious leaders, explaining the importance of mental health and appropriate mental health treatment. Religious leaders and places of worship play a key role in providing support, information, and spiritual leadership among African American communities. They are trusted messengers and influencers who often have a history of addressing health and mental health promotion. Places of worship increasingly leverage technology through radio broadcasts, Zoom sermons, Facebook Live, and podcasts. In churches, messages about seeking and receiving mental health treatment can be shaped by significant interpersonal social interactions (Gibbs et al., 2020).

Mental health care providers must remove the stigma associated with mental health treatment. One approach that can help reduce mental health treatment and illness stigmatization is education. Education strategies should be used to dispel commonly held myths regarding mental illness and treatment. Counselors must emphasize mental health promotion rather than mental illness treatment to reduce the overall stigma of mental health disorders. This strategy's goal should be to educate clients and the mental health clinicians and community (Maiden et al., 2021).

Finally, mental health care providers must acknowledge African Americans' mistrust of mental health care but also educate them of its importance. Discriminatory and exploitative behavior in medical research toward African Americans has led to understandable distrust among black communities. Counselors can address the distrust ab medical research promote the benefits of mental health care. This includes providing facts and data about COVID-19 and vaccination. Active communication with African American clients can lead to less emotional distress, depression, anxiety, and exclusion experiences.

\section{CONCLUSION}

The COVID-19 pandemic has affected the lives of all Americans, but some have been more adversely impacted. The social disruption and losses (whether to receive the vaccination, death of loved ones, Black Lives Matter Movement, and the elections, etc.) have generally impacted African Americans more severely than Whites due to a host of social factors that generate inequity in the United States. The longstanding effects of Racism and racist policies (bank loans, medical attention, racial profiling, etc.) must be addressed to improve African Americans' mental health and those with mental illness. The pandemic has highlighted these challenges and, with sustained efforts to fight Racism, may help lead our society to increased empathy and action to eradicate racial bias and disparity. Mental health care providers must ensure that critical messages about science, health, safety, and resources are communicated through trusted community networks and sources. Additionally, mental health care providers can help spread important information about health and vaccination. They can ensure that critical messages about science, health, safety, and resources are communicated through trusted community networks and sources.

\section{REFERENCES}

1. Buser, J. K. (2009). Treatment-seeking disparity between African Americans and whites: Attitudes toward treatment, coping resources, and racism. Journal of Multicultural Counseling \& Development, 37(2), 94104.

2. Centers for Disease Control and Prevention. (2022). Social Determinants of Health. Retrieved January 17, 2022, from https://www.cdc.gov/chronicdisease/ programs-impact/sdoh.htm

3. Cheatham, C. T., Barksdale, D. J., \& Rodgers, S. G. (2008). Barriers to health care and health-seeking behaviors faced by black men. Journal of the American Academy of Nurse Practitioners, 20(11), 555-562.

4. Cokley, K., Krueger, N., Cunningham, S. R., Burlew, K., Hall, S., Harris, K., Castelin, S., \& Coleman, C. (2021). The COVID-19/racial injustice syndemic and mental health among Black Americans: The roles of general and race-related COVID worry, cultural mistrust, and perceived discrimination. Journal of Community Psychology,1-20.https://doi.org/10.1002/jcop.22747

5. Cruz, M., Pincus, H. A., Harman, J. S., Reynolds, C.F., \& Post, E. P. (2008). Barriers to care-seeking for depressed African Americans. International Journal of Psychiatry in Medicine, 38(1), 71-80.

6. Diala, C.C., Muntaner, C., Walrath, C., Nickerson, K., \& LaVeist, T. (2001). Racial/Ethnic differences in attitudes toward seeking professional mental health services. American Journal of Public Health, 91(5) 805-807.

7. Frumkin, H. (2021). COVID-19, the built environment, and health. Environmental health perspectives, 129(7), 075001.

8. Gamble, V.N. Under the shadow of Tuskegee: African Americans and health care. American Journal of Public Health. (87) 1773-1778.

9. Getachew, Y., Zephyrin, L., Abrams, M.K., Shah, A., Lewis, C., \& Doty, M.M. (2020). Beyond the case count: The wide-ranging disparities of COVID-19 in the United States, The Commonwealth Fund. https://www. commonwealthfund.org/publications/2020/sep/ beyond-case-count-disparities-covid-19-united-states

10. Gibbs, T., Pauselli, L., Vieux,U., Solan,M. \& Rosenfield, P. (2020). Mental health disparities among Black Americans during the COVID-19 pandemic. Psychiatric Times, https://www.psychiatrictimes.com/view/ 
mental-health-disparities-among-black-americansduring-covid-19-pandemic

11. Goldstein, A. and Guskin, E. (2020). Almost one-third of black Americans know someone who died of covid19. Retrieved from https://www.washingtonpost. com/health/almost-one-third-of-black-americansknow-someone-who-died-of-covid-19-surveyshows/2020/06/25/3ec1d4b2-b563-11ea-aca5ebb63d27e1ff_story.html

12. Gould, E, and Wilson, V. (2020). Black workers face two of the most lethal preexisting conditions for coronavirusracism and economic inequality, Economic Policy Institute.

13. Healthy People 2030, U.S. Department of Health and Human Services, Office of Disease Prevention and Health Promotion. Retrieved January 16, 2022, from https:// health.gov/healthypeople/objectives-and-data/socialdeterminants-health

14. Ibrahimi, S., Yusuf, K. K., Dongarwar, D., Maiyegun, S. O., Ikedionwu, C., \& Salihu, H. M. (2020). COVID-19 Devastation of African American Families: Impact on Mental Health and the Consequence of Systemic Racism. International journal of MCH and AIDS, 9(3),390-393. https://doi.org/10.21106/ijma.408https://www.epi. org/publication/black-workers-covid/

15. Jackson, P., Brown, C., \& Callender, L. (2021). Healthrelated quality of life during stay-at-home order and attitudes toward vaccination against COVID-19 in the District of Columbia, USA. Journal of Health and Social Sciences, 6(1), 73-82.

16. Johnson, C. K., Rodriguez, O.R., and Kastanis, A. (2021). As US COIVD-19 death toll nears 600,000, racial gaps persist. Associated Press. Retrieved form https://apnews.com/article/baltimore-californiacoronavirus-pandemic-race-and-ethnicity-health341950a902affc651dc268dba6d83264
17. Kirby, T.(2020). Evidencemounts on the disproportionate effect of COVID-19 on ethnic minorities. The Lancet Respiratory Medicine, 8(6), 547-548.

18. Maiden, J. L., Mizelle, N., Nichols, B.J., \& Stewart, D.O. (2020). The impact of microaggressions on men of color in graduate counseling programs. International Journal of Social Policy and Education, 2(5).

19. Mizelle, N., Maiden, J.L. \& Stewart, D.O. (2020). Addressing racial disparities in mental health for African American males, International Journal of Humanities, Social Sciences and Education, 7(7), 199-205.

20. Noonan, A.S., Velasco-Mondragon, H.E., \& Wagner, F.A. (2016) Improving the health of African Americans in the USA: An overdue opportunity for social justice. Public Health Review, 37(12), 1-20.

21. Plowden, K. O. (2003). A theoretical approach to understanding black men's health-seeking behavior. Journal of Theory Construction \& Testing, 7(1), 27-31.

22. Scharff, D. P., Mathews, K. J., Jackson, P., Hoffsuemmer, J., Martin, E., \& Edwards, D. (2010). More than Tuskegee: understanding mistrust about research participation. Journal of health care for the poor and underserved, 21(3), 879-897.

23. Strauser, D.R., Ciftci, A., \& O’Sullivan, D. (2009). Using attribution theory to examine community rehabilitation provider stigma. International Journal of Rehabilitation Research. 32(1), 41-47.

24. U.S. Census Bureau, "Week 12 Household Pulse Survey: July 16-July 21," July 29, 2020. https://www. psychiatrictimes.com/view/mental-health-disparitiesamong-black-americans-during-covid-19-pandemic

25. U.S. Census. 2020. Household Pulse Survey: Measuring Social and Economic Impacts during the Coronavirus Pandemic. 2020. Retrieved January 16, 2022 from: https: / / www.census.gov/programs-surveys / household-pulse-survey.html

Citation: James Maiden, Phronie Jackson, Nathalie Mizelle, "COVID and African Americans: Addressing Disparities in Mental Health ", American Research Journal of Humanities and Social sciences, Vol 8, no. 1, 2022, pp. 26-30.

Copyright (c) 2022 James Maiden, Phronie Jackson, Nathalie Mizelle, This is an open access article distributed under the Creative Commons Attribution License, which permits unrestricted use, distribution, and reproduction in any medium, provided the original work is properly cited. 